\title{
Autism genetic testing: a qualitative study of awareness, attitudes, and experiences among parents of children with autism spectrum disorders
}

\author{
Lei-Shih Chen, PhD, CHES ${ }^{1}$, Lei $\mathrm{Xu}, \mathrm{MA}^{1}$, Tse-Yang Huang, $\mathrm{PhD}^{2}$ and Shweta U. Dhar, MD, MS ${ }^{3}$
}

\begin{abstract}
Purpose: The goal of this first-of-its-kind qualitative study was to examine the awareness, attitudes, and experiences among parents of autistic children regarding autism genetic testing.

Methods: We conducted in-depth, individual, and semistructured interviews with 42 parents of autistic children with diverse racial/ethnic backgrounds. All interviews were audio-taped, transcribed, and coded into major themes and subthemes.
\end{abstract}

Results: Approximately one-quarter of participants had two or more autistic children, and about half of them were ethnic/racial minorities. The majority of participants postulated favorable attitudes toward autism genetic testing for three main reasons: early intervention and treatment, identifying the etiology of autism, and informed family planning. Nevertheless, among parents who had taken their children

\section{INTRODUCTION}

The rapid growth of genomic medicine has led to dramatic advancements in identifying autism susceptibility genes, which in turn has increased the quality and quantity of genetic testing for autism spectrum disorders (ASD). ${ }^{1-3}$ According to the American Academy of Pediatrics ${ }^{4}$ and the American College of Medical Genetics and Genomics, ${ }^{5}$ the current standard ASD genetic testing for genetic diagnosis includes G-banded karyotyping and fragile $\mathrm{X}$ testing. The International Standards for Cytogenomic Arrays Consortium ${ }^{6}$ has recently recommended chromosomal microarray analysis (also known as array-comparative genomic hybridization), which has a better diagnostic yield, to replace current standard tests. The diagnostic yield of ASD genetic testing varies from $0.5 \%$ to $18 \%$, depending on the type of testing. ${ }^{7-10}$

Although genetic testing is now a standard of care and an integral part of evaluation of ASD, a genetic cause is identified in $<25 \%$ of patients with ASD. ${ }^{11}$ With the advancement of genomic technologies, another potential test-massively parallel sequencing (e.g., whole-exome and whole-genome sequencing analysis) - is gradually moving from the research laboratory to clinical settings for the identification of single genes associated with ASD. ${ }^{12-14}$ Therefore, it is expected that the genetic etiology of ASD will become more explicit in the future.

Generally, the potential benefits of ASD genetic testing include: (i) identifying the etiology of $\mathrm{ASD}^{2}$ (ii) promoting for genetic testing, some expressed frustration and questioned the competency of their providers in interpreting test results. Asian parents and those with a low socioeconomic status expressed lower awareness and tended to have more limited access to autism genetic testing.

Conclusion: As health-care providers play a vital role in providing genetic services and education, these professionals should be educated and be sensitive to the needs of parents with autistic children. Further quantitative research is required to examine the effects of socio-demographic factors on parents' awareness, attitudes, and experiences regarding autism genetic testing.

Genet Med 2013:15(4):274-281

Key Words: attitude, autism spectrum disorders, awareness, genetic testing, parents

early detection and intervention, ${ }^{2}$ (iii) developing treatment plans targeting ASD-associated medical conditions, ${ }^{15}$ and (iv) providing risk evaluation information for counseling and education for parents and families of affected children to empower them to make informed reproductive decisions. ${ }^{16}$ Nevertheless, in a similar way to other genetic tests, ASD genetic testing also raises many ethical, legal, and social issues. These encompass genetic discrimination, privacy concerns, psychological burden on the parents and families, and unequal access to genetic services. ${ }^{16-18}$ However, genetic testing for ASD faces more complex issues due to (i) the complexities involving ASD (e.g., ASD is a multifactorial disease that involves complex interactions between genes and environment, and manifests in multiple types and at various severity levels), (ii) challenges of ASD genetic testing (e.g., genetic causes cannot be identified in all autistic children), and (iii) attention from the mass media and general public. ${ }^{1,18,19}$

ASD genetic testing is often offered to individuals with a clinical suspicion of having this condition. The diagnosis of ASD is most commonly made during childhood. As children are unable to make responsible decisions on their own, the onus of deciding about testing usually lies with their parents or legal guardians. ${ }^{20}$ Perspectives and experiences regarding this testing among parents with autistic children, however, have not yet been addressed. To the best of our knowledge, this first-of-its-kind study has sought to examine the 
awareness, attitudes, and experiences of ASD genetic testing among parents with autistic children by answering the following questions: are parents with autistic children aware of ASD genetic testing? What are the attitudes of parents? What socio-demographic characteristics appear to shape their attitudes? And what are the underlying motivations and experiences among parents who have taken their children for ASD genetic testing?

\section{MATERIALS AND METHODS}

\section{Study design and participants}

To provide insights into perspectives regarding ASD genetic testing among parents of autistic children, we employed a qualitative study approach. Parents with at least one child diagnosed with ASD were eligible to participate. A snowball sampling technique was used to recruit a diverse sample of socio-demographic characteristics. An emergent design was adopted to permit refinement of the interview questions throughout various stages of data collection, analysis, and interpretation. ${ }^{21}$ This study was approved by Texas A\&M University's Institutional Review Board.

\section{Interview guide}

On the basis of previous literature, ${ }^{22-24}$ we developed a semistructured interview guide. Six experts in ASD, genetics/ genomics, public health, special education, and/or qualitative research reviewed and revised the interview questions. To establish a rapport with participants and understand their background, interviews started with a general question asking parents to describe their experiences with their autistic children. Interviewees were then asked to elicit in-depth responses regarding five topics: (i) perceived causes and recurrence risk of ASD; (ii) awareness, attitudes, and experiences regarding ASD genetic testing; (iii) utilization of genetic services; (iv) family planning; and (v) genetic/genomic education needs. In this study, we focused on parental views and experiences regarding ASD genetic testing. Supplementary Figure S1 online illustrates the interview flowchart.

\section{Data collection}

We disseminated recruitment letters and approached potential participants through eight large autism parent groups/ programs and four professional conferences in Texas. To avoid redundancy and repetitiveness, ${ }^{21}$ participants were interviewed until data reached theme saturation. The first two authors conducted the majority of the interviews ( $n=40 ; 95 \%)$, while the remaining two were conducted by a graduate assistant. Half of the interviews $(n=21)$ were carried out face-to-face, and the rest were done either by telephone $(n=16)$ or Skype live-cam $(n$ $=5$ ). Thirty-one interviews were in English and 11 in Chinese/ Taiwanese. Interviews lasted for $\sim 1 \mathrm{~h}(\mathrm{SD}=1.4 \mathrm{~h}$; range $=52$ min-2 h). All participants were requested to sign written consent forms and were paid US\$50 as an incentive.

To ensure data quality and interview consistency, the first two authors frequently met to discuss the data and revised the interview guide as emerging themes developed. All interviews were recorded digitally and transcribed into English or Chinese, depending on the language spoken during interview. Field notes were taken during interviews, which were later used to supplement the transcriptions for further interpretation and elucidation of the data. ${ }^{21}$ A brief social-demographic survey was used to collect the information on participants and their families.

\section{Data analysis}

Using Nvivo 8.0 software (QSR International Pty, Doncaster, Australia) to facilitate the coding process and compare reoccurring themes across the transcription data, the first two authors of this manuscript (L.S.C. and L.X.) employed content analysis to code and discuss the themes. When divergent coding emerged, the transcription was reviewed again to reach an agreement. We also further examined the potential differences in identified themes among different socio-demographic subgroups. A research team in the field of genomics and public health (one member was an autistic patient) reviewed and discussed the coding themes with the authors. This investigator triangulation contributed to analytic robustness and ensured multiple perspectives for the findings of the study. ${ }^{21}$

\section{Sample characteristics}

\section{RESULTS}

Supplementary Table S1 online shows our sample, which comprised 42 parents with at least one child diagnosed with ASD. The majority of participants had one autistic child, while approximately one-quarter had two or more autistic children. The majority were mothers (76\%), half of whom were at reproductive age. About half of the participants were white, and the other half were Asian (26\%), Hispanic (14\%), black (7\%), and mixed (2\%).

\section{Interview findings}

Emerged themes and illustrative quotations from the interview data are provided in Tables $\mathbf{1 - 4}$. The details are described as follows.

Parents' awareness of ASD genetic testing. Nineteen (63\%) of 30 parents who had not taken their children for ASD genetic testing reported that they had never heard of this test before the interview. For example, one Asian parent asked us, "What test did you say? Genetic testing for autism? I've never heard of it before. It sounds like science fiction to me." The remaining 11 parents were aware of this testing, but most had obtained this information from mass media and scientific articles $(n=$ 5 ), and communication with autism support groups and other parents of autistic children $(n=4)$. Only two interviewees learned about ASD genetic testing from their children's doctors (Table 1).

White parents with a high socioeconomic status (SES) tended to be more familiar with ASD genetic testing. None of the parents who had an annual household income $<\$ 35,000$, or had a 


\begin{tabular}{|c|c|c|}
\hline Theme & Sources of information & Illustrative quotation \\
\hline $\begin{array}{l}\text { Heard of ASD } \\
\text { genetic testing }\end{array}$ & $\begin{array}{l}\text { Recommendations from } \\
\text { health-care providers (e.g., } \\
\text { pediatricians, geneticists) }\end{array}$ & $\begin{array}{l}\text { "Well, this developmental pediatrician said to us, why don't you do a genetic test? I } \\
\text { recommend a genetic test, blah, blah, blah, for your future children." (mother, white) } \\
\text { "I've heard of genetic testing, they [doctors] are trying to considering do genetic } \\
\text { testing for fragile X." (mother, Latino) }\end{array}$ \\
\hline
\end{tabular}

ASD, autism spectrum disorders.

high school diploma or less had heard of ASD genetic testing. Asian parents belonged to the lowest awareness group: $70 \%$ of them had never heard about this test.

Parents' attitudes toward ASD genetic testing. We asked participants to express their opinions regarding ASD genetic testing. Most $(n=29 ; 69 \%)$ postulated favorable attitudes, while approximately one-quarter $(n=11 ; 26 \%)$ were against this testing, and two parents (5\%) held a neutral position. The sections below explain the underlying reasons for parents' responses.

\section{In favor of ASD genetic testing}

Table 2 lists parents' reasons for supporting ASD genetic testing, ranging from the highest to the lowest frequencies. Early intervention and treatment was the most frequently identified reason $(n=15)$. For instance, a mother with one autistic and one normally developing child said she would like to learn about ASD genetic testing because this information would help her affected child to receive early intervention, resulting in a better outcome. In addition, 13 parents supported genetic testing because they would like to find the etiology of ASD. These parents perceived genetics as the contributing factor to ASD. For example, one mother with a high SES stated, "I personally support genetic testing for autism...especially seeing the [ASD] percentage go[ing] up makes me think that...somebody is genetically susceptible." Another father with a low SES remarked, "I think that it's a good development. We are trying to figure out how DNA works to help us understand our
bodies...I might know why I had kids with autism from it. I think it is useful."

Eight parents believed that ASD genetic testing might help them make informed family planning decisions. One white mother with three autistic children (the eldest died at the age of 13) told us "I'm sure [sighs] there is something with me genetically ... because with two different husbands, I've had all my biological children with ASD." This participant adopted a normally developed child as her fourth child. Another 32-yearold Asian mother commented, "If the ASD genetic test result is positive, she [her daughter with autism] can decide to adopt a child instead of having a child of her own." One white mother strongly believed that all women should undergo ASD genetic testing for making reproductive decisions, stating, "I think all young ladies need to be tested by the time they are 16 years old so they would know if they have the genes that would cause them to have children with autism." Finally, other participants were in favor of ASD genetic testing for the reasons of helping with research $(n=2)$, eliciting more family support $(n=1)$, and assistance with health insurance coverage $(n=1)$.

\section{Against ASD genetic testing}

About one-quarter of parents $(n=11)$ opposed ASD genetic testing. Among these parents, the majority did not consider genetics as a causal factor for ASD. We further asked parents their reasons for opposing ASD genetic testing. Six participants articulated that they did not foresee its value because: (i) in their view ASD was incurable and the test 
Table 2 Reasons for supporting ASD genetic testing among parents with at least one child with ASD $(n=29)$

Theme

Early intervention

and treatment

etiology of ASD

Illustrative quotation

"If you find out that your child has autism right when they are born, then you can start intervention from day one. There are children who do get cured and lead more normal lives." (mother, white)

"A genetic testing of a newborn should be the same as a 70 years old, so, why not get that information earlier? Why not do something about it instead of waiting till it is too late?" (mother, Asian)

"I support [ASD] genetic testing as long as it will not harm my baby, and I think it is valuable and should be done because it does prepare you for the right person to go to and the right treatment." (father, Latino) "I think that they should test you when you first get pregnant, because if they can do the test for Down syndrome, they can test for autism ahead of time. And then you know where you have to go, and what you are going to deal with, when you give birth and all things that come with it. You are prepared." (mother, black)

(mother, black)

\begin{abstract}
"I am very receptive to [ASD] genetic testing. I am a pragmatist, so, genetic testing, certainly in our case, a blessing because we know where it came from." (father, white)

"I think genetic testing can help us to know the causes (of autism). We can try this or that, however, without knowing the etiology, all the treatments are blind...finding the causes can also help to alleviate the family burden and benefit the whole society." (mother, Asian)

"My opinion is, it is like so many unanswered questions, I think genetic testing is a way that we can unlock lots of mysteries... think it is a much better route." (father, black)
\end{abstract}

Informed family planning

"Biologically, it is relevant in terms of reproduction. Because if there is a genetic component to autism in my family, then it would give me pause to think about having another child." (mother, white)

"I think it is beneficial to my daughter [with autism]. If genetic testing is positive, she could choose to adopt a child when she grows up." (mother, Asian)

Supporting ASD research

"If you want to do genetic testing as part of research, trying to identify genes or something like that. I have no problem with that." (father, white)

"It would be interesting...It certainly wouldn't do anything for us, but for purpose of research I would do it. We've done this from the very beginning..." (mother, white)

Eliciting more

family support

"Mostly to get my support group lined up. To get everybody on board versus yes he does, or no, he doesn't need this, he doesn't need that, because our family is very caring and concerned and they slammed me with the things I need to be doing for him. I am like 'you wanna help?' It is 7 AM and you gave me quite the list." (mother, white)

"I think if I can have this genetic testing done, then I can be more affirmative in asking the health insurance company to pay the bill for the treatment." (mother, white)

ASD, autism spectrum disorder.

would not improve their current situation; (ii) their children had already been diagnosed with ASD or had grown up; (iii) it was too late to have gene therapy for their affected children; (iv) it would not make any difference in their affected children's lives; and (v) they did not plan to have any future pregnancies or had no family history. Another four parents believed that ASD genetic testing does more harm than good. They were afraid that overemphasizing the genetic factor of ASD would not only overlook other nongenetic causes but also stigmatize the affected families. Furthermore, they were afraid their health/life insurance would be declined or become more expensive if the test results were positive.

Contradictions with religious or cultural beliefs were mentioned by three interviewees. Specifically, two mothers with Christian beliefs perceived that the notion of genetic testing was against God's will. The other Asian father articulated, "I think I must have committed something terrible in my previous life. How come I am the only one with an autistic child? I don't think genetic testing can do anything now. I have to accept it all as my destiny."

Two parents stated that genetic testing might cause family conflicts. It might become a blame game between the couple, once the test results identified whose genes contributed to the child's symptoms. One parent questioned the existence and method of ASD genetic testing itself (Table 3).

\section{Neutral attitudes}

Two parents acknowledged that there were advantages and disadvantages of ASD genetic testing, and were waiting for more evidence in this research area to make their judgment.

Parents' willingness of taking their children to undergo ASD genetic testing. We asked parents whose children had not undergone ASD genetic testing $(n=30)$ whether they would be willing to take their children for this test. Nearly half of them ( $n$ $=14$ ) expressed their interest. Supporting ASD research was the most frequently cited reason $(n=8)$. According to one mother, "It certainly would not do anything for us, but I would support it for research." Elucidating the etiology of their children's ASD was another reason disclosed by four parents. The other interviewee thought that the test results might help their autistic children make informed family planning, once the children grow up.

Conversely, $38 \%$ of the interviewees ( $n=11$ ) would refuse to take their children to be tested. For example, one parent with 
Table 3 Reasons for opposing ASD genetic testing among parents with at least one child diagnosed with ASD $(n=11)$

Theme

Perceiving no value of ASD genetic testing for affected children and their families

Contradicting religious/ cultural beliefs

Causing family conflicts

Questioning the existence and method of ASD genetic testing
Illustrative quotation

"I mean it [ASD genetic testing] would make no difference, it would make absolutely no difference in his (the autistic son's) programming or placement. It would make no difference in the way the school interacted with him, it would make no difference in any of the way he lived..." (mother, white)

"It would be worthwhile to get the genetic test done at an earlier age when gene therapy would be helpful. I just don't think it would be helpful right now." (mother, white)

"I really don't think it is necessary to do it at this moment ... she had been diagnosed with autism anytime, it was too late to undergo genetic testing." (mother, Asian)

"He [the autistic son] is the only one with autism. I have 6 cousins and their children are all healthy. We just do not have a family history of autism. I do not know how genetic testing can be helpful in my case." (mother, black)

Doing more harm than good

"I think it does more harm than good. I think it is trying to mask looking at other causes. It is already putting a burden on those who have a burden. I think that it is an easy way out for the medical community to say it is genetic." (mother, white)

"... because there are issues of getting insurance. If you have genetic testing and something is found, people's health insurance and life insurance will be declined, or more expensive." (mother, Latino)

"I do not want to do it [ASD genetic testing]. My daughter lives in her own world. But so what? She is a gift from God. I do not want to challenge God's will." (mother, Asian)

"I don't want to play the blame game. I cannot change who [child's name omitted for confidentiality] is. It won't do any good. It would only do her good for later on. But for me, I was already dealing with it so what was the point. It wasn't going to change her if I found out who was to blame for, you know, who had it and who did not ..." (mother, white)

"I do not believe the test methods. If someone really can figure it out, then they can win the Nobel Prize. It is just like so many factors contributing for people to get cancer. Someone said some medicine or food lead to cancer, someone talks about curse ... autism has such a broad range, it is impossible to find fixed genes that cause this tendency, I wish it could be that simple ..." (father, Asian)

ASD, autism spectrum disorder.

Table 4 Underlying motivations in having their autistic children undergo ASD genetic testing among parents with at least one autistic child $(n=12)$

Theme

Hoping to find genetic

cause of ASD

Receiving recommendations

from health-care providers

\section{Illustrative quotation}

"I remembered he [the child with autism] did the test [ASD genetic testing] when he was six or seven years old. We wanted to know if it is a hereditary factor that caused his autism." (mother, Asian) "I wanted to see which one of us [husband and I] contributed to her [autistic daughter's] autism, and to know why the cell is not working right." (mother, Asian)

"We went to see a pediatric neurologist. He recommended that we test for some common genetic anomalies. He [child with autism] was tested for fragile X..." (mother, white)

"They [pediatric providers] did complete genetic screens on them [two children with autism]...they thought [child's name omitted for confidentiality] might have Angelman [syndrome] ..." (mother, white)

Supporting ASD research

"I know my daughter had fragile $X$ testing before and that was negative. We did it [genetic testing] for supporting research." (mother, white)

Getting influenced by family members

Finding out other health

problems

"My husband wanted her [daughter with ASD] to do this test, I really don't." (mother, Asian)

"We [husband and I] wanted to rule out, especially like fragile X syndrome that is common with autism, and autism is a part of it and just his karyotype with it, at that time. Just to make sure we couldn't find like any other health problem with him [child with ASD]." (mother, black)

Looking for better treatment

"A better treatment and a better understanding..." (mother, Latino)

ASD, autism spectrum disorder.

an 11-year-old autistic child remarked, "Well, we actually were not given that option for ASD genetic testing before. I know it sounds funny. But earlier on, we could have embraced it a lot more. But at this stage, I do not see any use in it." In addition, one low-income Chinese American mother pointed out transportation as a barrier.

Finally, four parents of high-functioning autistic children were reserved in their decision during the interview. They would prefer to wait for their children to grow up and make their decisions themselves.

Parents' motivations and experiences of taking children for ASD genetic testing. Table 4 illustrates the underlying motivations of 12 parents (two-thirds had a high SES) who had taken their children for ASD genetic testing. Regarding testing experiences, one-third expressed their frustration. According to one 
Latino mother, "In [name of hospital omitted for confidentiality] hospital, the process is very long. You have to fill out lots of forms, you have to go through lots of testing, and you have to see a neuropsychologist and a psychologist. And so, by the time all that was done, they finally said, 'why don't you get some genetic testing?' And I was thinking, it would be nice to have had that in the first place. We already spent a whole year."

In addition, these four parents also questioned their providers' competency in interpreting test results. For example, a three-year-old daughter of an Asian mother recently underwent ASD genetic testing. Based on the mother's experience, she was frustrated as she could not understand the genetic/ medical terms in the report and none of the health professionals addressed her concerns at that time. She even requested for our research team's assistance in interpreting the report during the interview.

\section{DISCUSSION}

To the best of our knowledge, this is the first-of-its-kind study assessing the awareness, perspectives, and experiences of ASD genetic testing among parents of autistic children. Instead of recruiting participants from hospitals, who had access to health care, we obtained our sample from various ASD communities. We used a qualitative approach because it allowed for in-depth elicitations of unexamined research domains, helped to facilitate understanding of new genetic technology adoptions, and contributed to the clarification of this complex topic. ${ }^{21,25}$

This study showed numerous important findings. First, most participants were in favor of ASD genetic testing. Early intervention and treatment, elucidation of the etiology, and informed family planning were the main factors supporting their decision. However, approximately one-quarter of the parents were against ASD genetic testing and expressed their concerns regarding its value, method, harms, and religious/ cultural conflicts. Some of their concerns, such as genetic discrimination, privacy issue, and psychological burden on families, were consistent with the literature discussing the ethical, legal and social implication of ASD genetic testing. ${ }^{16}$ As parents hold different attitudes, policymakers should thoroughly consider the perspectives of both parties when developing relevant regulations and recommendations. For example, should health-care providers recommend all parents with ASD children to take their affected children for such testing? How do we ensure that testing results will benefit the affected families without causing them further harm and/or burden? Moreover, although the Genetic Information Nondiscrimination Act (GINA) ${ }^{26}$ preventing health insurance companies from denying coverage to individuals who have already undergone genetic testing was passed in May 2008, there is still limited public awareness regarding this Act. ${ }^{27}$ Therefore, many families may choose to forgo genetic testing for fear of losing the much needed health insurance benefits. Increasing awareness about GINA among both health-care providers and parents may reduce the concern of discrimination and privacy regarding genetic testing.
Second, ASD genetic testing has been offered for more than a decade in medical practice. ${ }^{5}$ Nevertheless, the majority of the participants, who had not taken their children for ASD genetic testing, had never heard about such testing. This finding is in line with the previous study-the lay public has insufficient awareness of genetic testing. ${ }^{28}$ According to the Autism A.L.A.R.M. Guidelines ${ }^{29}$ proposed by the American Academy of Pediatrics and Centers for Disease Control and Prevention, physicians should "educate parents and provide them with upto-date information." Therefore, health-care providers should make their patients and families aware of the availability of ASD genetic testing. Furthermore, although the lay public has deficient awareness of genetic testing, this is also the case with health-care providers. ${ }^{30,31}$ As such, genomic education for health-care providers, along with the lay public, is also needed to improve the overall awareness and understanding of ASD genetic testing among both parties.

Several misunderstandings regarding ASD genetic testing emerged during the interviews. Some parents believed that genetic testing could not be useful to families once their children were diagnosed with ASD or had grown up. Moreover, some thought that this testing is beneficial only for parents with a family history of ASD or parents who are planning to have future pregnancies. These findings are consistent with past research which indicated that lay people might not fully comprehend or misunderstand genetic testing. ${ }^{28,32}$ Given that these misperceptions might subsequently affect parents' testing decisions, reproductive planning, and treatment planning for their autistic children, health-care providers should address these issues to facilitate better health outcomes for affected children and families.

Notably, this study revealed that few participants learned about ASD genetic testing from their health-care providers. Among parents who had taken their children for ASD genetic testing, some had negative experiences and low confidence in their providers' ability to interpret test results. This finding is supported by past research which showed that doctors had inadequate competencies to discuss the medical condition with the families of children with developmental delays. ${ }^{33-35}$ In addition, studies have also found that health-care providers have limited genomic competencies for discussing genetic testing with patients. ${ }^{30,31}$ Accordingly, genomics education for health-care providers is needed to facilitate better genetic services.

Of note, when asking parents' willingness in taking their children for ASD genetic testing, aiding ASD research was a most frequently mentioned reason. This finding has been previously noted in other studies. ${ }^{36-38}$ For example, in Chen and Goodson's systematic review study, ${ }^{36}$ one-fifth of the reviewed articles revealed research-related factors affecting individuals' acceptance of cystic fibrosis carrier testing. Similarly, other studies have also found that assistance and support for research motivate people to undergo $B R C A 1 / 2$ genetic testing. ${ }^{37,38}$

Finally, as in other studies, ${ }^{20,39}$ our data suggested disparities in the awareness and access of ASD genetic testing among this 
sample. Interviewees with a low SES tended to be less familiar with and had less access to this testing. Asian participantsmostly immigrants with a low SES-constituted the group expressing the lowest awareness of ASD genetic testing. To minimize genomics-related health disparities, genomics education and relevant support for parents with a low SES or immigrant status is needed.

This study suffered from several limitations. Past studies have suggested that socio-demographic characteristics might influence individuals' perceptions regarding genetic testing. ${ }^{20,32,40}$ For example, Hudson et al. ${ }^{40}$ noticed that different socio-demographic groups (e.g., race/ethnicity, income, and education level) had various awareness levels and opinions concerning genetic testing. Therefore, we purposely recruited participants with different socio-demographic statuses. Although half of the interviewees were racial/ethnic minorities, most had a high SES. This sample might have higher genetic knowledge than the general public. In addition, although parents' experiences might vary on the basis of the type of ASD genetic tests their children had undertaken, most could not state the test type. Future studies should be conducted to examine parents' testing experiences regarding different types of ASD genetic testing. Furthermore, health-care providers should clearly communicate the type of testing to parents of autistic children before their children undergo the actual test procedure. Finally, all participants seem to be relatively well connected with the specific autism community to which they are affiliated. The findings of this study, therefore, may not be representative of all the parents of autistic children.

\section{Conclusions}

This qualitative study provides insight into medical practice, health policies, and future research directions regarding ASD genetic testing. Although most parents of autistic children supported this testing, they seemed to have low awareness/ knowledge regarding ASD genetic testing. Advocating for better health education for both parents of autistic children and the public regarding availability, types, advantages, and disadvantages of ASD genetic testing is needed. Moreover, among those who had taken their children for this testing, one-third had a negative experience. As pediatric providers play a vital role in providing genetic services and education, these professionals should be educated and be sensitive to the needs of parents with autistic children. For example, training healthcare providers to enhance their communication skills in explaining the procedure and findings regarding ASD genetic testing may facilitate better patient-provider relationships. Furthermore, policymakers should consider parents' attitudes to make appropriate recommendations. Finally, as compared with other groups, Asian parents and those with low SES expressed lower awareness and tended to have more limited access to autism genetic testing. Future quantitative research is required to examine the effects of socio-demographic factors on parents' awareness, attitudes, and experiences regarding ASD genetic testing.

\section{ACKNOWLEDGMENTS}

This study was funded through Lei-Shih Chen's grant-Texas A\&M University's Program to Enhance Scholarly and Creative Activities. We thank Tung-Sung Tseng, Laura Stough, Elsa M. Gonzalez y Gonzalez, Corey Parshall, Allison Hollek, Eunju Jung, and Champa Joshi for their assistance with this study.

\section{DISCLOSURE}

The authors declare no conflict of interest.

\section{REFERENCES}

1. Abrahams BS, Geschwind DH. Advances in autism genetics: on the threshold of a new neurobiology. Nat Rev Genet 2008;9:341-355.

2. Johnson HM, Gaitanis J, Morrow EM. Genetics in autism diagnosis: adding molecular subtypes to neurobehavioral diagnoses. Med Health $R I$ 2011;94:124-126.

3. Miller DT. Genetic testing for autism: recent advances and clinical implications. Expert Rev Mol Diagn 2010;10:837-840.

4. Myers SM, Johnson CP; American Academy of Pediatrics Council on Children With Disabilities. Management of children with autism spectrum disorders. Pediatrics 2007;120:1162-1182.

5. Schaefer GB, Mendelsohn NJ; Professional Practice and Guidelines Committee. Clinical genetics evaluation in identifying the etiology of autism spectrum disorders. Genet Med 2008;10:301-305.

6. Miller DT, Adam MP, Aradhya S, et al. Consensus statement: chromosomal microarray is a first-tier clinical diagnostic test for individuals with developmental disabilities or congenital anomalies. Am J Hum Genet 2010;86:749-764.

7. Sebat J, Lakshmi B, Malhotra D, et al. Strong association of de novo copy number mutations with autism. Science 2007;316:445-449.

8. Shen Y, Dies KA, Holm IA, et al.; Clinical genetic testing for patients with autism spectrum disorders. Pediatrics 2010;125:e727-e735.

9. Roesser J. Diagnostic yield of genetic testing in children diagnosed with autism spectrum disorders at a regional referral center. Clin Pediatr (Phila) 2011;50:834-843.

10. Reddy KS. Cytogenetic abnormalities and fragile-X syndrome in Autism Spectrum Disorder. BMC Med Genet 2005;6:3.

11. Gurrieri F. Working up autism: the practical role of medical genetics. Am $J$ Med Genet C Semin Med Genet 2012;160C:104-110.

12. O'Roak BJ, Deriziotis $P$, Lee $C$, et al. Exome sequencing in sporadic autism spectrum disorders identifies severe de novo mutations. Nat Genet 2011:43:585-589.

13. Voineagu I, Wang $X$, Johnston $P$, et al. Transcriptomic analysis of autistic brain reveals convergent molecular pathology. Nature 2011;474:380-384.

14. Mefford HC, Batshaw ML, Hoffman EP. Genomics, intellectual disability, and autism. N Eng/ J Med 2012;366:733-743.

15. Filipek PA, Accardo PJ, Ashwal S, et al. Practice parameter: screening and diagnosis of autism: report of the Quality Standards Subcommittee of the American Academy of Neurology and the Child Neurology Society. Neurology 2000;55:468-479.

16. Marchant GE, Robert JS. Genetic testing for autism predispostion: ethical, legal, and soical challenges. Houst J Health Law Policy 2009:203-235.

17. Jordan BR, Tsai DF. Whole-genome association studies for multigenic diseases: ethical dilemmas arising from commercialization-the case of genetic testing for autism. J Med Ethics 2010;36:440-444.

18. Skinner D, Sparkman KL, Bailey DB Jr. Screening for Fragile X Syndrome: parent attitudes and perspectives. Genet Med 2003;5:378-384.

19. Whitman T. The Development of Autism: A Self-regulatory Perspective. London, England: Jessica Kingsley Publishers; 2004

20. Campbell E, Ross LF. Parental attitudes and beliefs regarding the genetic testing of children. Community Genet 2005;8:94-102.

21. Lincoln YS, Guba EG. Naturalistic Inquiry. Thousand Oaks, CA: Sage Publications;1985.

22. Selkirk CG, McCarthy Veach P, Lian F, Schimmenti L, LeRoy BS. Parents' perceptions of autism spectrum disorder etiology and recurrence risk and effects of their perceptions on family planning: Recommendations for genetic counselors. J Genet Couns 2009;18:507-519. 
23. Miquel-Verges F, Woods SL, Aucott SW, Boss RD, Sulpar LJ, Donohue PK. Prenatal consultation with a neonatologist for congenital anomalies: parental perceptions. Pediatrics 2009;124:e573-e579.

24. Mercer L, Creighton S, Holden JJ, Lewis ME. Parental perspectives on the causes of an autism spectrum disorder in their children. J Genet Couns 2006;15:41-50.

25. Barbara $A B$. The role of qualitative research in medicial genetics: listening to the voices of our patients. Am J Med Genet. Part A 2008;146A:3132-3135

26. Genetic Information Nondiscrimination Act (GINA) of 2008. <http://www gpo.gov/fdsys/pkg/PLAW-110publ233/pdf/PLAW-110publ233.pdf>. Accessed 3 October 2012.

27. Allain DC, Friedman S, Senter L. Consumer awareness and attitudes about insurance discrimination post enactment of the Genetic Information Nondiscrimination Act. Fam Cancer 2012.

28. Lanie $A D$, Jayaratne $T E$, Sheldon JP, et al. Exploring the public understanding of basic genetic concepts. J Genet Couns 2004;13:305-320.

29. Centers for Disease Control and Prevention. Autism A.L.A.R.M. Guidelines 2010 <http://www.medicalhomeinfo.org/downloads/pdfs/AutismAlarm. pdf $>$. Accessed September 8, 2012.

30. Baars MJ, Henneman L, Ten Kate LP. Deficiency of knowledge of genetics and genetic tests among general practitioners, gynecologists, and pediatricians: a global problem. Genet Med 2005;7:605-610.

31. Freedman AN, Wideroff $L$, Olson $L$, et al. US physicians' attitudes toward genetic testing for cancer susceptibility. Am J Med Genet Part $A$ 2003;120A:63-71.
32. Chen L, Zhao M, Zhou Q, Xu L. Chinese Americans' views of prenatal genetic testing in the genomic era: a qualitative study. Clin Genet 2012;82:22-27.

33. Kemper AR, Bailey DB Jr. Pediatricians' knowledge of and attitudes toward fragile X syndrome screening. Acad Pediatr 2009;9:114-117.

34. Golnik A, Ireland M, Borowsky IW. Medical homes for children with autism: a physician survey. Pediatrics 2009;123:966-971.

35. Harrington JW, Patrick PA, Edwards KS, Brand DA. Parental beliefs about autism: implications for the treating physician. Autism 2006; 10:452-462

36. Chen LS, Goodson P. Factors affecting decisions to accept or decline cystic fibrosis carrier testing/screening: a theory-guided systematic review. Genet Med 2007;9:442-450.

37. Phillips KA, Warner E, Meschino WS, et al. Perceptions of Ashkenazi Jewish breast cancer patients on genetic testing for mutations in BRCA1 and BRCA2. Clin Genet 2000;57:376-383.

38. Hallowell N, Foster C, Ardern-Jones A, Eeles R, Murday V, Watson M. Genetic testing for women previously diagnosed with breast/ovarian cancer: examining the impact of BRCA1 and BRCA2 mutation searching. Genet Test 2002;6:79-87.

39. Catz DS, Green NS, Tobin JN, et al. Attitudes about genetics in underserved, culturally diverse populations. Community Genet 2005;8:161-172.

40. Hudson K, Scott J, Brauch S et al. Reprodutive Genetic Testing: What America Thinks. 2004a. <http://www. dnapolicy.org/pub.reports. php?action=detail\&reprot_id=6>. Accessed 8 September 2012. 